\title{
Effect of Physical Exercise on Autonomic Nerve Function in Irritable Bowel Syndrome
}

\author{
Karma Tenzin ${ }^{1}$, Noorzahan Begum ${ }^{2}$, Sultana Ferdousi ${ }^{3}$
}

\begin{abstract}
Background: Irritable bowel syndrome (IBS) may be associated with autonomic nerve dysfunction. However, moderate to vigorous physical exercise cause improvement of this nerve function. Objective: To observe the effect of moderate physical exercise on the autonomic nerve activity by analysis of HRV in patients with Irritable bowel syndrome. Methods: This prospective study was carried out in the Department of Physiology, Bangabandhu Sheikh Mujib Medical University (BSMMU), Dhaka in 2013. For this purpose, 77 male patients aged 20-50 years with IBS were included in the study. They were subdivided into IBS-D (24 patients), IBS-C (26 patients) and IBS-A (27 patients) and were selected from the OPD, Gastroenterology, BSMMU. 28 apparently healthy male were studied as control. HRV parameters were recorded prior to onset of physical exercise and after completion of 3 months of brisk walking. For assessing autonomic nerve function, some time domain measures of HRV were recorded by Polyrite D. ANOVA, Independent sample t-test and paired t-tests were used for statistical analysis. Results: The pre-exercise values of mean R-R interval, Max/Min ratio, SDNN,RMSSD, NN50\%, PNN50\% were significantly $(\mathrm{p}<0.05)$ lower and mean heart rate was significantly $(\mathrm{p}<0.05)$ higher in all IBS patients compared to control. The post exercise data showed mean HR was significantly decreased in IBS-A and SDNN \& RMSSD were significantly increased in IBS-C and IBS-A. Conclusion: This study concluded that parasympathetic activity decreased in patients with IBS, which was improved after regular moderate physical exercise of 3 months.
\end{abstract}

Key words: Irritable bowel syndrome, Autonomic nerve function, Male, Physical exercise, Heart rate variability.

\section{Introduction}

I rritable bowel syndrome (IBS) takes up a major bulk in gastrointestinal associated health issues in most developed countries with a prevalence rate of $10-20 \%{ }^{1}$ and also equally common in developing countries ${ }^{2}$. IBS is a chronic continuous or remittent functional gastro-intestinal disorder that is characterized by various signs and symptoms including abdominal

Received Jan. 2014;

Accepted May 2014 pain, bloating and bowel disturbance ${ }^{3}$. It has been classified by Rome II criteria as Diarrhoea predominant irritable bowel syndrome (IBS-D), Constipation predominant IBS (IBS-C) and Irritable bowel syndrome alternating type (IBSA $)^{4}$. Diarrhoea predominant IBS was the dominant IBS subgroup and the age range of these patients was 15 to 44 years ${ }^{5}$.

Recently IBS has been reported as a disorder of the brain gut axis, involving abnormal function

J Bangladesh Soc Physiol. 2014, June; 9(1): 17-21 
at the enteric, autonomic and central nervous system level. Association of vagal dysfunction in IBS-C whereas sympathetic dysfunction with IBS-D has been reported by some investigators whereas no discrimination of dysfunction among the subtypes was also reported ${ }^{2,4}$.

Improvement of autonomic nerve function following regular physical exercise have been reported in healthy adolescent and in working class and also in menopause ${ }^{6-13}$.

Heart Rate Variability analysis(HRV) is a powerful tool for assessing autonomic nerve function 16 Increased HRV reflects good adaptability and well functioning autonomic nerve control but reduced HRV can predict risk for adverse CVS events in broad range of diseases ${ }^{17}$.Mean R-R interval and the mean heart rate, Max/min ratio, SDNN, RMSSD, NN50), and pNN50 are used for HRV measurement as time domain measures ${ }^{16}$.Lower values of R-R interval, SDNN, RMSSD, PNN50\% and NN50\% and higher heart rate were noted in IBS patients 2,4 .

Physically active subjects displayed higher mean R-R interval, SDNN, RMSSD and lower mean heart rate ${ }^{7,8}$ and physical inactivity ${ }^{18}$ associated decreased in HRV were noted in IBS patients ${ }^{2}$. Though, few investigators reported of improvement of HRV with physical activity in healthy subjects ${ }^{7-8}$, are available but evidence of this effect in IBS patients are lacking.

Therefore, this study aimed to evaluate the effect of moderate physical exercise on cardiac autonomic nerve activity in patients with IBS by analysis of heart rate variability.

\section{Methods}

This prospective intervention study was conducted in the Department of Physiology, Bangabandhu Sheikh Mujib Medical University (BSMMU), Dhaka from January to December 2013. The protocol of this study was approved by Institutional Review Board of BSMMU. 77 male aged 20-50 years, diagnosed case of Irritable bowel syndrome were enrolled for the study by random sampling from Gastroenterology Department of BSMMU, Dhaka. According to the predominant symptom ${ }^{1}$, IBS patients were divided into 3 subgroups including 24 diarrhea predominant IBS (IBS-D), 26 constipation predominant IBS (IBS-C) and 27 altered bowel habit type IBS (IBS-A) patients. Age and BMI matched 28 apparently healthy sedentary male were also enrolled as control. History of heart disease, diabetes mellitus, hypertension, chronic renal failure, thyroid disorders and smoking were excluded and also history of regular physical exercise were ruled out in all selected patients. Informed written consent was taken from each subject. Subsequently, a detail family and medical histories were taken to rule out any chronic and known illness which runs in the family and also physical activity status were recorded.

For HRV recording, the subjects were prepared from one day prior to the test. They were advised to finish their meal by 9:00 p.m and to have sound sleep and not undergo any sort of physical or mental stress, and were also advised to avoid taking any sedatives or any drugs affecting central nervous system in the night before the test. The patient was advised to take light breakfast in the morning without tea and coffee. All the examinations were done in the Autonomic Nerve Function Test Laboratory in the Department of Physiology, BSMMU. For the purpose of HRV recording, the subject was given bed rest for 15-20 minutes, after which 5 minutes ECG recording was carried out for HRV by RMS polyrite D. Data of IBS patients were recorded once before exercise then once after completion of 3 months of exercise. Controls were sedentary and they did not undergo any kind of physical exercise. HRV data of control were recorded once.

The IBS patients performed regular moderate physical exercise (brisk walking) for 3 months. The patients were trained for brisk walking at a rate of 130 steps per minute ${ }^{20}$ for 30 to 45 minutes, for 3 to 5 days in a week for a period of 3 months duration $24,26$.

J Bangladesh Soc Physiol. 2014, June; 9(1): 17-21 
Mean R-R interval, mean heart rate, $\mathrm{max} / \mathrm{min}$ ratio, SDNN, RMSSD, PNN50\% and NN50\% were recorded and analyzed before exercise and similar procedure was also adopted after 3 months of exercise. Data were then compared between pre and post exercise value in all IBS patients. ANOVA, independent t-test and paired t-test were used for statistical analysis. P value $<0.05$ was taken as level of significance.

\section{Results}

The pre-exercise values of systolic blood pressure (SBP), diastolic blood pressure (DBP) and pulse rate $(\mathrm{PR})$ were found to be significantly higher in all IBS patients (Table I)The pre-exercise values of heart rate was significantly higher and mean R-R interval, SDNN, RMSSD, PNN50\% and NN50\% were significantly lower all IBS groups than those of the control. No significant differences of these parameters were observed within subgroups of IBS. (Table II)

Table I: General Characteristics in different groups $(\mathrm{n}=105)$

\begin{tabular}{lcccc}
\hline Parameters & Control & IBS-D & IBS-C & IBS-A \\
\hline Age & $32 \pm 1.26$ & $32 \pm 1.36$ & $29 \pm 1.36$ & $32 \pm 1.37$ \\
BMI & $22.8 \pm 0.29$ & $22.2 \pm 0.48$ & $21.8 \pm 0.43$ & $22.3 \pm 0.44$ \\
SBP & $114 \pm 1.55$ & $120 \pm 1.63 *$ & $120 \pm 1.96 *$ & $120 \pm 1.79 *$ \\
DBP & $71 \pm 1.25$ & $75 \pm 1.59 *$ & $77 \pm 1.66 *$ & $76 \pm 1.24 *$ \\
PR & $74 \pm 0.96$ & $86 \pm 1.64 * *$ & $84 \pm 1.23 * *$ & $86 \pm 1.48^{* *}$ \\
\hline
\end{tabular}

Values in means \pm SE for Diarrhoea predominant Irritable bowel syndrome (IBS-D), Constipation predominant Irritable bowel syndrome (IBS-C) \& Altered bowel habit type of Irritable bowel syndrome $(*=$ control vs IBS ** $\mathrm{p}<0.01 \& * \mathrm{p}<0.05)$

Table II: Pre and post exercise frequency domain measures of HRV in different groups ( $\mathrm{n}=105)$

\begin{tabular}{|c|c|c|c|c|c|c|c|c|}
\hline \multirow[t]{2}{*}{$\begin{array}{l}\text { HRV } \\
\text { measures }\end{array}$} & \multicolumn{2}{|c|}{ Control } & \multicolumn{2}{|c|}{$\begin{array}{c}\text { IBS-D } \\
\text { (Exercise) }\end{array}$} & \multicolumn{2}{|c|}{$\begin{array}{c}\text { IBS-C } \\
\text { (Exercise) }\end{array}$} & \multicolumn{2}{|c|}{$\begin{array}{c}\text { IBS-A } \\
\text { (Exercise) }\end{array}$} \\
\hline & Baseline a & fter 3months & 1s Pre & Post & Pre & Post & Pre & Post \\
\hline $\mathrm{R}-\mathrm{R}$ interval & $0.78 \pm 0.02$ & $0.79 \pm 0.02$ & $0.69 \pm 0.02 *$ & $0.73 \pm 0.03$ & $0.69 \pm 0.02 *$ & $0.72 \pm 0.02$ & $0.68 \pm 0.02 * * *$ & $0.73 \pm 0.02$ \\
\hline Mean HR & $73 \pm 1.35$ & $71 \pm 1.59$ & $85 \pm 3.60 *$ & $80 \pm 1.83$ & $86 \pm 2.06^{* *}$ & $80 \pm 2.12$ & $83 \pm 1.90 * *$ & $77 \pm 1.84 \dagger$ \\
\hline Max/Min ratio & $1.79 \pm 0.1$ & $1.77 \pm 0.1$ & $2.04 \pm 0.16$ & $1.82 \pm 0.11$ & $1.66 \pm 0.09^{\#}$ & $1.74 \pm 0.08$ & $1.57 \pm 0.1^{\# \#}$ & $1.73 \pm 0.07$ \\
\hline SDNN & $82 . \pm 3.90$ & $83.5 . \pm 2.9$ & $46 \pm 2.70 * *$ & $52.8 \pm 3.2$ & $45.9 \pm 2.90 * *$ & $56.4 \pm 2.7 \ddagger$ & $48.5 \pm 2.40 * *$ & $63.5 \pm 2.70 t t$ \\
\hline RMSSD & $39.2 \pm 2.8$ & $40.2 \pm 1.7$ & $29.01 \pm 2.8^{*}$ & $37.0 \pm 3.3$ & $29.74 \pm 2.5^{*}$ & $38.0 \pm 2.1 \ddagger$ & $30.6 \pm 2.1 *$ & $38.2 \pm 1.7+t$ \\
\hline PNN50\% & $21.9 \pm 2.8$ & $20.7 \pm 1.9$ & $9.6 \pm 0.9 * *$ & $12.9 \pm 2.2$ & $8.2 \pm 0.8 * *$ & $10.9 \pm 1.3$ & $11.6 \pm 1.6^{* *}$ & $12.3 \pm 0.8$ \\
\hline NN50\% & $65 \pm 5.40$ & $62 \pm 4.20$ & $35 \pm 3.6 * *$ & $42 \pm 4.2$ & $34 \pm 3.7 * *$ & $39 \pm 4.6$ & $39 \pm 5.8^{* *}$ & $40 \pm 5.1$ \\
\hline
\end{tabular}

Values are means \pm SE, Statistical analysis were performed by independent sample t-test and paired t-test. $(* * \mathrm{p}$

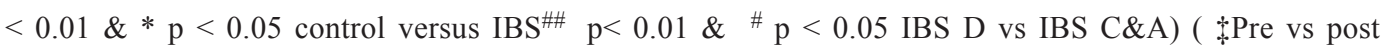
exercise $(\$ \mathrm{p}<0.5 ; \$ \mathrm{p}<0.01)$. IBS-D $=$ Diarrhoea Predominant IBS-C $=$ Constipation predominant IBS-A = Altered bowel habit type IBS

The post exercise heart rate was significantly decreased only in IBS-A. whereas post exercise SDNN and RMSSD were significantly increased only in IBS-C and IBS-A $\mathrm{c}$ than their corresponding preexercise values. (Table-II). 


\section{Discussion}

This present study assessed the change in autonomic nerve function activity in IBS patients induced by moderate physical exercise. Present data suggested reduced HRV in IBS patients before exercise compared to healthy person which agrees to our previous study ${ }^{2}$.

Significantly lower values of R-R interval, SDNN, RMSSD, PNN50\% and NN50\% in all IBS subgroups suggests lower parasympathetic modulation in all IBS patients which is comparable to others. ${ }^{2,4,21,22}$.Parasympathetic dysfunction in IBS-C is accountable to the development of constipation predominance ${ }^{4}$. However findings of the present study did not show any such discrimination regarding parasympathetic activities among the subtypes of IBS.

After 3 months of brisk walking the IBS patients showed significant improvement of autonomic nerve function indicated by significantly higher post exercise values of the parameters than their pre-exercise values. This result agrees to the findings of some of the studies who observe the effect of physical exercise in healthy individuals 7-8. Despite this improvement the autonomic function remain away from that of healthy person demonstrated by significant difference of the post exercise data from that of a healthy person.

Max/min ratio in IBS-C and IBS-A showed reduced variability than IBS-D before exercise but this result after exercise did not reflect any significant variation between different subtypes. Though, the overall variability was improved in all IBS patients but this improvement failed to show any discrimination among the subgroups.

moderate physical exercise is associated with either increased vagal tone or decreased sympathetic modulation of heart rate in both healthy and diseased conditions 9,$19 ; 23$.Physical exercise reduces the stress by influencing brain plasticity 24 which results in higher parasympathetic activity and cardiovagal baroreflex sensitivity. In addition, both acetylcholine and choline-acetyl transferase content in cardiac tissue may be increased ${ }^{8}$. Regular physical exercise also might cause attenuation of cardiac beta-receptor activity and decline in norepinephrine concentration which causes decrease in sympathetic nerve activity. In addition, this exercise promotes adjustments in cardiovascular control sites via neural remodeling or factors like nitric oxide and angiotensin II. These adaptations cause decrease in sympathetic and increase in vagal component in cardiac control to improve cardiac autonomic balance ${ }^{25-26}$. The improvement of autonomic function may be linked to the effect of physical exercise.

\section{Conclusion}

This study concluded that patients of all 3 subgroups of IBS had noticeably decreased parasympathetic activities and moderate physical exercise increased their cardiac parasympathetic activity.

\section{Authors affiliation:}

1. Dr. Karma Tenzina, MD Student, Department of Physiology, BSMMU, Dhaka, E-mail: karmat enenzina9@gmail.com

2. Dr. Noorzahan Begum, Professor, Department of Physiology, BSMMU, Dhaka

3. Dr. Sultana Ferdousi, Sultana Ferdousi, Associate Professor, Department of Physiology, BSMMU, Dhaka

\section{References}

1. Thompson WG, Heaton KW. Functional bowel disorder in apparently healthy people. Gastroenterology. 1980;79:283-8.

2. Nayem M, Begum N, Ferdousi S. Time domain of heart variability to assess autonomic dysfunction in irritable bowel syndrome. J. Bangledesh Soc Physiol. 2012; 7(2): 60-65.

3. Clark C, DeLegge M. Irritable bowel syndrome :a practical approach. Nutr Clin Pract. 2008; 23: 263-267.

4. Heitkemper M, Jarrett M, Cain KC, Burr R, Levy RL, Feld A, Hertig V. Autonomic nervous system function in women with irritable bowel syndrome. Digestive Disease and Sciences. 2001; 46(6): 12761284 .

5. Perveen I, Hasan M, Masud MA, Rahman MM, Bhuiyan MMR. Irritable bowel syndrome in a

J Bangladesh Soc Physiol. 2014, June; 9(1): 17-21 
Bangladeshi Urban community: Prevalance and Health care seeking pattern. The Saudi journal of Gastroentrology. 2009; 15(4): 239-43.

6. Costa PJ, Carvalho H, Freitas A, Ramos J, Puga N, Lomba I, Fernandes P, de Freitas F. Spectrum analysis of the variability heart rate in athletes. Rev Port Cardiol. 1991;10(1): 23-8.

7. Alom MM, Begum N, Naher LAD, Faruky SB, Akter N. Study on heart rate variability in adolescent male athletes by time domain (Short-Term) method. The ORION Medical Journl. 2010; 33(1): $722-725$

8. Mithun S, Begum N, Ferdousi S, Begum S, Ali T. Time domain measures of heart rate variability in heavy workers. J Bangladesh Soc Physiol. 2009:6(2): 77-83

9. Malfatto G, Facchini M, Bragato R, Branzi G, Sala L, Leonetti G. Short and long-term effects of exercise training on the tonic autonomic modulation of heart rate variability after myocardial infarction. Eur Heart J. 1996; 17: 532-538.

10. Iellamo F, Pizzinelli P, Massaro M, Raimondi G, Peruzzi G, Legramante JM. Muscle metaboreflex contribution to sinus node regulation during static exercise: Insight from spectral analysis of heart rate variability. Circulation. 2000; 100: 27-32.

11. Pagkalos M, Koutlianos N, Kouidi E, Pagakalos E, Mandroukas K, Deligiannis A. Heart rate variability modifications following exercise training in type 2 diabetic patients with definite cardiac autonomic neuropathy. Br J Sports Med. 2008; 42: 47-54.

12. Dina C, Rebsburg JV, Ker JA, Grants CC, Fletcher L. Effect of exercise on cardiac autonomic function in females with rheumatoid arthritis. Clin Rheumatol. 2012; 31: 1155-1162.

13. Jurca R, Church TS, Morss GM, Jordan AN, Earnest CP. Eight weeks of moderate intensity exercise training increases heart rate variability in sedentary post-menopausal women. Am Heart J. 2004;147(5): e21.

14. Bonaduce D, Petretta M, Cavallaro V, Apicella C, Ianniciello A, Romano M, Breglio R, Marciano F. Intensive training and cardiac autonomic control in high level athletes. Med Sci Sports Exerc. 1998; 30(5): 691-696.

15. Figueroa A, Baynard T, Fernhall B, Carhart R, Kaneley JA. Endurance training improves postexercise cardiac autonomic modulation in obese

J Bangladesh Soc Physiol. 2014, June; 9(1): 17-21 women with and without type-2 diabetes. Eur J Appl Physiol. 2007; 100: 437-444.

16. Task Force of the European Society of Cardiology and the North American Society of pacing and electrophysiology. Heart Rate Variability. standards of measurement, physiological interpretation and clinical use. Circulation. 1996; 93: 1043- 65.

17. Evrengül H, Dursunoglu D, Cobankara V, Polat B, Seleci D, Kabukeu S, Kaftan A, Semiz E, Kilic M. Heart Rate Variability In Patients With Rheumatoid Arthritis. Rheumatol Int. 2004;24: 198-202.

18. Dong Y, Zuo X, Li C, Yu Y, Zhao Q, Li Y. Prevalence of irritable bowel syndrome in Chinese college and university students assessed using Rome III criteria. world J Gastroenterol. 2010;16(33): 4221-26.

19. Du N, Bai S, Oguri K, Kato Y,Matsumoto I, Kawase $\mathrm{H}$, Matsuoka T. Heart rate recovery after exercise and neural regulation of heart rate variability in $30-40$ year old female marathon runners. J of sports science and medicine. 2005; 4: 9-17.

20. Hancock C. Review: The benefits of physical activity for health and well-being. C3 collaborating for Health. 2011 [Cited 2014, Jan]. Available from: http://www. msse.org.

21. Jarrett ME, Burr RL, Cain KC, Hertig V, Weisman P, Arnp M, Heitkemper MM. Anxiety and depression are related to autonomic nervous system function in women with irritable bowel syndrome. Digestive Diseases and Sciences. 2003; 48(2): 386-394.

22. Tillisch K, Mayer EA, Labus JS, Stains J, Chang L, Naliboff BD. Characterization of the alternating bowel habit subtype in patients with irritable bowel syndrome. Am J gastroenterol. 2005; 100: 896-904.

23. Marcos B A, Araujo CG. Effects of aerobic training on heart rate. Rev Bras Med Esporte. 2003; 9: 113-120.

24. Johannesson E, Simren M, Strid H, Bajor A, Sadik R. Physical activity improves symptoms in irritable bowel syndrome: a rantomized controlled trial. Am J Gastroenterol. 2011;106: 915-922.

25. Marud K, Brubaker PH, Fitzgerald DM, Morgan TM, Goff DC, Soliman EZ, Eggebeen JD, Kitzman DW. Exercise training improves heart rate variability in older patients with heart failure: a randomized. Controlled, single-blinded trial. Congest heart fail. 2012;18: 192-197.

26. Miyashita M, Burns SF, Stensel DJ. Accumulating short bouts of brisk walking reduce postprandial plasma triacylglycerol concentrations and resting blood pressure in healthy young men. Am J Clin nutr. 2008; 88: 1225-31. 